\title{
Primary Thyroid-Like Follicular Carcinoma of the Kidney: A Cases Report with Detailed CT Radiology Profile
}

\author{
Di Yu and Yan Lei* \\ Shandong University of Qilu hospital, China \\ Received: 制 June 21, 2018; Published: 䟧July 11, 2018 \\ *Corresponding author: Yan Lei, Shandong University of Qilu hospital, China
}

\begin{abstract}
Primary thyroid-like follicular carcinoma of the kidney (PTLFC) is an unusual histological variant of renal cell carcinoma not included in the current WHO classification of renal tumors. Our paper illustrate the histological characteristics of vacuolar degeneration and CT signs of the case. The masses has the characteristic of Calcification, lower density than the renal cortex and stronger than the necrosis areas, its enhancement $(65.33 \pm 14.66 \mathrm{HU})$ has a moderate degree than the cortex $(94.63 \pm 19.33 \mathrm{HU})$ and make a frosted glasses shadow in the solid areas. ECT suggests low perfusion index and hypometabolism. These signs may help the diagnose of the disease before the operation.
\end{abstract}

Keywords: Renal Cell Carcinoma; Thyroid-Like Follicular Carcinoma; CT Signs

\section{Introduction}

A 32-year-old Chinese woman got mild trauma and made physical examination, she has a past medical history of twice cesarean section operation. A massive space occupying lesions was found in the right kidney and sent to the Urology Department of Qilu Hospital for further treatment in January 2017. The abdominal ultrasound showed the right kidney mass of confounding echo. The CT scan revealed a round moderately hypo-dense and wellvascularized solid mass which is about $18 \times 13 \times 9 \mathrm{~cm}$ with a necrotic central area near the renal cortex, the number of lymph nodes near the aortic area increases without any density abnormity. After other examination and radiology tests, an Laparoscopic radical right nephrectomy was carried to the patient. The pathology suggests an Thyroid-like follicular carcinoma of the kidney. And then, a series tests of the thyroid was done,this include B-ultrasound scan, CT of the neck,T3,T4,thyroid immuno-globulins in blood-serum and so on, all the results excludes any lesions in her thyroid.Moreover, it can showed that the tumor was not a metastatic disease. She was discharged the hospital with good post-operative recovery. The cancer did not reappear in the follow-up visits after her surgery. Before the operation, she has low serum Retinol binding protein $(18.7 \mathrm{mg} / \mathrm{L})$ and creatinine clearance ratio $34 \mathrm{umol} / \mathrm{L})$;her $\beta$-hydroxy butylate $(0.59 \mathrm{mmol} / \mathrm{L})$ and $\operatorname{ESR}(67.00 \mathrm{~mm} / \mathrm{h})$ were increased. Her CT characteristics are shown as (Figure1)and (Table 1), which includes characteristic of the calcification,necrosis,contrast agent fast in and out, enhanced degree less than the cortex reins with a ground-glass shadow.Thekidneyandits perirenalfatisabout18 $\times 13 \times 9 \mathrm{~cm}$, therenalcapsuleiseasilytobestripped. Thecolorofthetumorisacommixofgreyyellow ,grey red, grey black, greywhite, its border is clear, therenalpelvis is in involvement. The renal cortex is about $0.5 \mathrm{~cm}$ thick and the renal medullary is about $1.5-2.5 \mathrm{~cm}$. The pathologypicturesanditsfeaturesareasfollows (Figure 2) and (Table 2).

Table 1: Tumor's Features on CT value, solid tumor components' Enhancement degree is about $65.33 \pm 14.66 \mathrm{HU}$, lower than the renal cortex $94.63 \pm 19.335 \mathrm{HU}$.

\begin{tabular}{|c|c|c|c|}
\hline & Precontrast $(\mathbf{H U})$ & Aftercontrast $($ HU) & Enhancement Degree $($ HU) \\
\hline Necrosis area in the tumor & $37.43 \pm 9.034$ & $41.04 \pm 12.21$ & $3.61 \pm 10.622$ \\
\hline Solid tumor components & $38.87 \pm 10.86$ & $104.2 \pm 18.46$ & $65.33 \pm 14.66$ \\
\hline Renal cortex & $41.27 \pm 10.38$ & $135.9 \pm 28.29$ & $94.63 \pm 19.335$ \\
\hline aorta & $46.18 \pm 10.09$ & $285.9 \pm 10.71 \mathrm{~s}$ & $239.72 \pm 10.4$ \\
\hline liver & $62.2 \pm 6.144$ & $81.29 \pm 6.762$ & $19.09 \pm 6.453$ \\
\hline
\end{tabular}


Table 2: Two kidney function data on ECT, the right kidney function area is about $33 \mathrm{~cm}^{\wedge} 2$ lower than the left one, so as the perfusion index and up take ratio, that means the tumor affects the renal function.

\begin{tabular}{|c|c|c|}
\hline Kidney & Left & Right \\
\hline Kidney area $\left(\mathrm{cm}^{\wedge} 2\right)$ & 84.4 & 49.23 \\
\hline Kidney depth $(\mathrm{cm})$ & 4.83 & 3.86 \\
\hline Perfusion index & 68.24 & 26.76 \\
\hline Uptake\% & 73.08 & 20.51 \\
\hline GFR & 56.27 & 20.48 \\
\hline Time to peak & 5.48 & \\
\hline
\end{tabular}

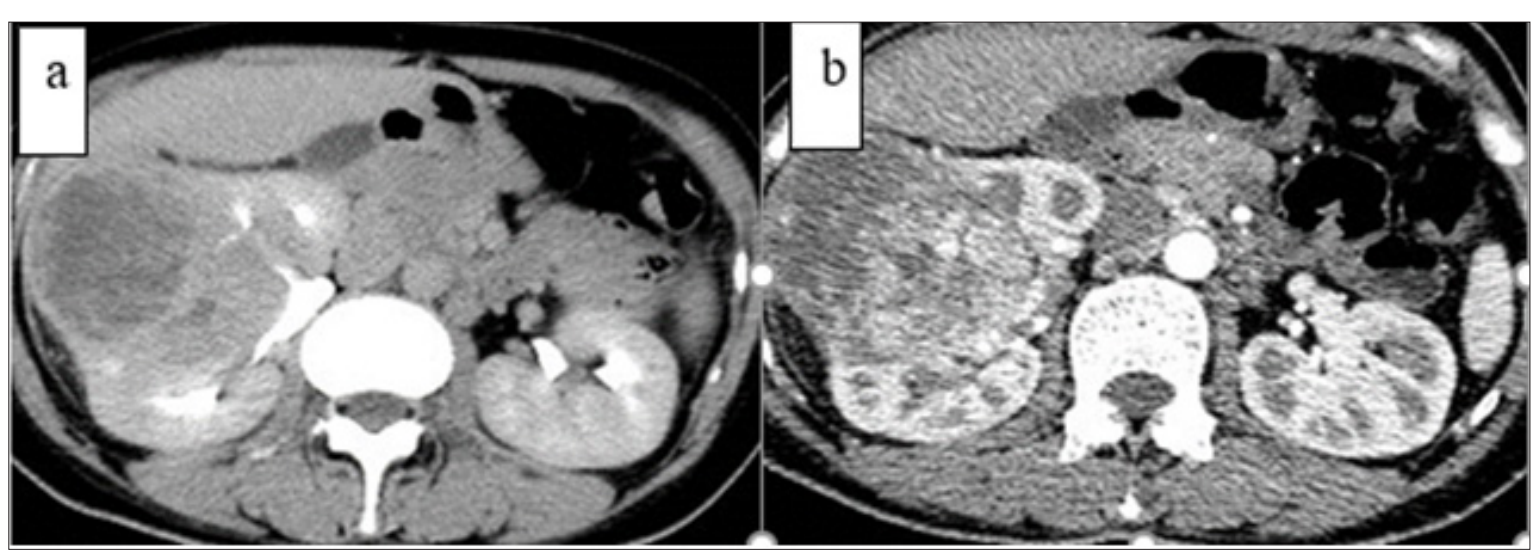

a) The area around the calcification is low density necrosis. The solid parts of the tumor has a slightly higher density than the necrosis' and lower than the renal cortex's. Its margins is irregular and do not have an obvious border to the normal tissues. When undergoing the enhanced CT scan, the tumor has an thick rim of enhancement and the degree is lower than the renal cortex. The necrosis inside the tumor do not have any change, the thinner renal cortex is mildly enhanced for the reason of compression by the tumor.

b) The solid portion of the mass is enhanced as frosted glass and invades into the renal pelvis. What is more, the superior renal vessel is also besen affected. The area around the aorta near the renal hilum has increased lymph gland node with the similar enhancement to the solid tumor.

Figure 1: The CT film findings of the patient display two calcification places.

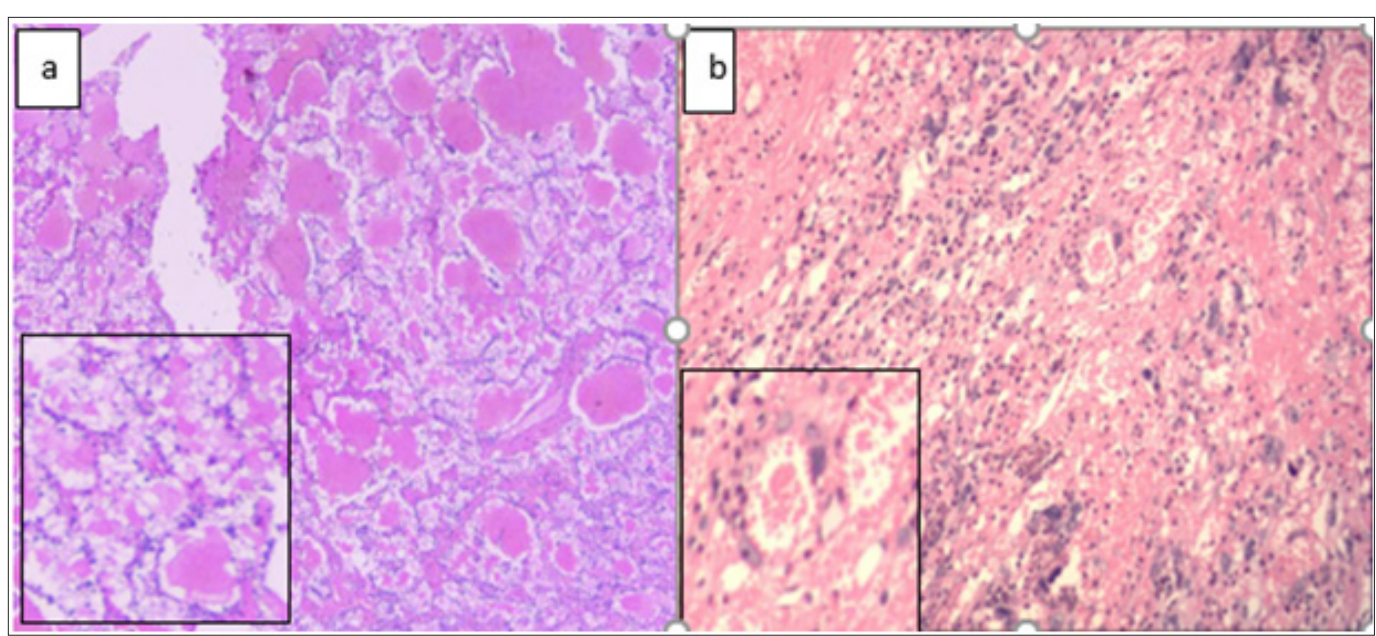

a. Follicular-like structures of thyroidgland is surrounded by basophilic tumor cells stained deeply by nuclei. The tumor epithelial cells are seen commonly of multiple vacuolar degeneration.

b. The parts of the tumor invading the renal pelvis:it has a nephron structure, the visible part of glomerular cysts is widen , glomerular is smaller and its capillary has not any nuclear architectures. Mesenchymal tissue is filled with colloid-like material in atrophic distal tubules and collecting ducts and polymorphic cell, whose nuclei is greater variability in sizes and appearances.

Figure 2: Follicular architecture construct with macro- and microfollicles filled with colloid-like material(HE). 


\section{Discussion}

PTLFC,occurring commonly in young patients, is a rare disease in renal carcinoma mimicing follicular carcinoma[1], and it is not included in the current WHO histological variant classification and has a debate within the pathologists' circles[2]. Its morphological features resemble a large spectrum of renal and extra-renal diseases which should be keeping out in the diagnostic process[3,4].It is distinguished by atrophic distal tubules and collecting ducts with colloid-like material in the optical microscope[5]. Compared with other renal tumors, this type of tumor has no specific location and clinical symptoms. Limited data showed that the prognosis of the tumor is good, but case accumulation and long-term follow-up are still needed[6]. Diagnosis of the disease should exclude renal metastasisofthyroid follicular carcinoma, renal primary Carcinoid or neuroendocrine carcinoma, and Epithelial Wilms tumor[7]. Renal metastasis of cancerous goiter is a rare event, with only few cases described, its primary lesion is easy to find and often accompany with widespread neoplastic diffusion[8]. Renal primary Carcinoid or neuroendocrine carcinoma rarely havefollicular structures and red staining colloid in the renal tubules and collecting ducts[9], As reported by literature, PPM1D,CDC27,SGOL2,PARD3,KIF25, MLL, DLG5, RBBP6, CLASP1, TFDP2 are over-expressed and RHOB,CBLB,ZFYVE16,RAB7L1 are under expressed compared to the chromophobe renal cell carcinoma. Carcinoid tumor cell has a smaller nuclei and more delicate Chromatin; necrosis is seldom seen in general. The atypia of neuroendocrine carcinoma cell is more obvious, so is the karyokinesis[10].

Epithelial Wilms tumor is a rare disease in children, almost not reported in adults. The epithelial cell constructs a chrysanthemumlike shape; tubular and papillary structures are sometimes visible[11]. But follicular structure with colloid colloid has not been reported. The histopathology of the disease is detailly illustrated by pre-reported cases, but their radiology scan has not been introduced, especially the CT aspects[12].The CT film displayed that calcification is a significant sign of the disease with low density necrosis surrounded. The masses has the characteristic of Calcification, lower density than the renal cortex and stronger than the necrosis areas, its enhancement $(65.33 \pm 14.66 \mathrm{HU})$ has a slighter degree than the cortex $(94.63 \pm 19.33)$ and make a frosted glasses shadow in the solid areas. ECT suggests low perfusion index and hypometabolism, which affects the renal function. Multiple vacuolar degeneration is seen in the HE pathologic picture in our case, and its affection to the glomerular and renal tubules has been described. In conclusion, the CT sign of the PTFLC pointed out by our studies may be significant differential points to the preoperative diagnosis.

\section{References}

1. Alaghehbandan R, Michal M, Kuroda N (2017) Thyroid-like Follicular Carcinoma of the Kidney. International Journal of Surgical Pathology 25(1): 73-77.

2. Cao D (2013) Thyroid-like follicular carcinoma of the kidney. Journal of Urology 190(1): 278-279.

3. Amin MB, Gupta R, Ondrej H, McKenney JK, Michal M, et al. (2009) Primary thyroid-like follicular carcinoma of the kidney: report of 6 cases of a histologically distinctive adult renal epithelial neoplasm. American Journal of Surgical Pathology 33(3): 393-400.

4. Dhillon J, Mohanty SK, Krishnamurthy S (2014) Cytologic diagnosis of thyroid-like follicular carcinoma of the kidney: a case report. Diagnostic Cytopathology 42(3): 273-277.

5. Ghaouti M, Roquet L, Baron M, Christian Pfister, Jean Christophe Sabourin (2014) Thyroid-like follicular carcinoma of the kidney: a case report and review of the literature. Diagnostic Pathology 9(1): 186.

6. Dong L, Huang J, Huang L,Oumin Shi, Qiang Liu, etal. (2016) Thyroid-Like Follicular Carcinoma of the Kidney in a Patient with Skull and Meningeal Metastasis: A Unique Case Report and Review of the Literature. Medicine 95(15): 3314.

7. Insabato L, BenDor D, Galliani CA, Lastilla G, Bisceglia M (2009) Primary thyroid and thyroid-like follicular carcinoma of the kidney versus solitary metastatic carcinoma of the thyroid: a vexing issue. VirchowsArchiv An International Journal of Pathology 454(6): 717-718.

8. Jung SJ, Chung JI, Park SH, Ayala AG, Ro JY (2006) Thyroid follicular carcinoma-like tumor of kidney: a case report with morphologic, immunohistochemical, and genetic analysis. American Journal of Surgical Pathology 30(3): 411-415.

9. Sterlacci W, Verdorfer I, Gabriel M (2008) Thyroid follicular carcinomalike renal tumor: a case report with morphologic, immunophenotypic, cytogenetic, and scintigraphic studies. Virchows Archiv An International Journal of Pathology 452(1): 91-95.

10. Muscara MJ, Simper NB, Gandia E (2016) Thyroid-Like Follicular Carcinoma of the Kidney: An Emerging Renal Neoplasm with Curiously Misplaced Histologic Features; a Case Report. International Journal of Surgical Pathology 25(1): 73.

11. Dawane R, Grindstaff A, Parwani AV, Timothy Brock, Wesley M White, et al. (2015) Thyroid-Like Follicular Carcinoma of the Kidney: One Case Report and Review of the Literature. American Journal of Clinical Pathology 144(5): 796-804.

12. He CN, Li P, Zhao HF,Zhai JP, Liu YQ et al. (2008) Thyroid follicular carcinoma-like tumor of kidney: report of a case. Zhonghua Bing LI Xue Za Zhi Chinese Journal of Pathology 37(6): 428-430.

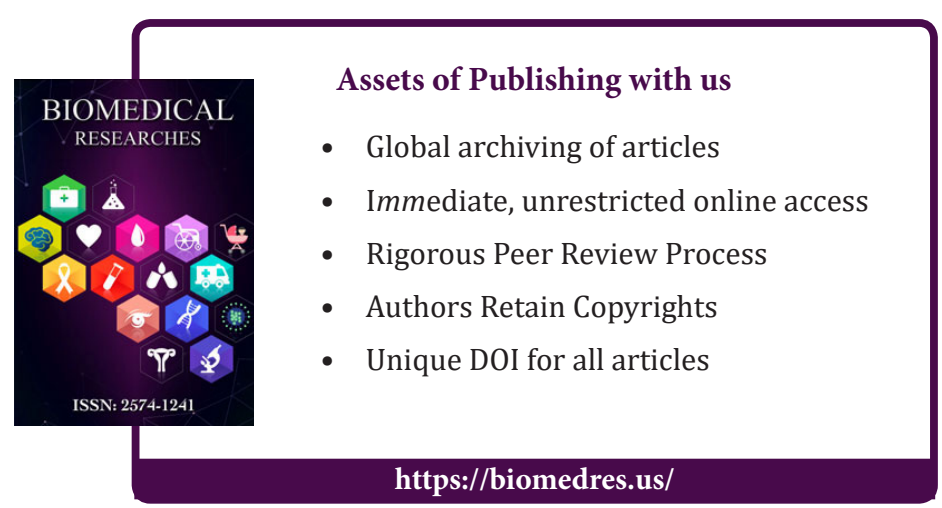

\section{ISSN: 2574-1241}

\section{DOI: 10.26717/BJSTR.2018.06.001385}

Di Yu and Yan Lei. Biomed J Sci \& Tech Res

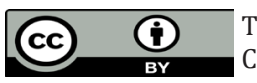

This work is licensed under Creative Commons Attribution 4.0 License

Submission Link: https://biomedres.us/submit-manuscript.php 